\title{
A Genome-Wide Association Study of Resistance to Puccinia striiformis f. sp. hordei and P. graminis f. sp. tritici in Barley and Development of Resistant Germplasm
}

\author{
Javier Hernandez, ${ }^{1}$ Alicia del Blanco, ${ }^{2}$ Tanya Filichkin, ${ }^{1}$ Scott Fisk, ${ }^{1}$ Lynn Gallagher, ${ }^{2}$ Laura Helgerson, ${ }^{1}$ Brigid Meints, ${ }^{1}$ \\ Chris Mundt, ${ }^{3}$ Brian Steffenson, ${ }^{4}$ and Patrick Hayes ${ }^{1, \dagger}$ \\ ${ }^{1}$ Department of Crop and Soil Science, Oregon State University, Corvallis, OR 97331 \\ 2 Department of Plant Sciences, University of California-Davis, Davis, CA 95616 \\ ${ }^{3}$ Department of Botany and Plant Pathology, Oregon State University, Corvallis, OR 97331 \\ ${ }^{4}$ Department of Plant Pathology, University of Minnesota, St. Paul, MN 55108 \\ Accepted for publication 3 February 2020.
}

\begin{abstract}
Stripe rust (incited by Puccinia striiformis f. sp. hordei) and stem rust (incited by $P$. graminis f. sp. tritici) are two of the most important diseases affecting barley. Building on prior work involving the introgression of the resistance genes rpg4/Rpg5 into diverse genetic backgrounds and the discovery of additional quantitative trait locus (QTLs) for stem rust resistance, we generated an array of germplasm in which we mapped resistance to stripe rust and stem rust. Stem rust races TTKSK and QCCJB were used for resistance mapping at the seedling and adult plant stages, respectively. Resistance to stripe rust, at the adult plant stage, was determined by QTLs on chromosomes $1 \mathrm{H}, 4 \mathrm{H}$, and $5 \mathrm{H}$ that were previously

selected as potentially resistant to stem rust or susceptible but having other positive attributes, showed resistance at the seedling stage, which appears to be allelic to rpg4/Rpg5. The rpg4/Rpg5 complex, and this new allele, were not sufficient for adult plant resistance to stem rust in one environment. A QTL on 5H, distinct from Rpg5 and a previously reported resistance QTL, was required for resistance at the adult plant stage in all environments. This QTL is coincident with the QTL for stripe rust resistance. Germplasm with mapped genes/QTLs conferring resistance to stripe and stem rust was identified and is available as a resource to the research and breeding communities.
\end{abstract} reported in the literature. The rpg4/Rpg 5 complex was validated as a source of resistance to stem rust at the seedling stage. Some parental germplasm,
Keywords: barley, multi-rust resistance, stem rust, stripe rust
Barley (Hordeum vulgare) is an ancient crop, with production beginning some 10,000 years ago (Badr et al . 2000). Today, barley is the fourth most important cereal crop in the world (FAOSTAT 2017) and is grown in a wide range of environments (Hayes et al. 2003). A versatile crop with multiple end-uses, barley is essential for the manufacturing of malt and beer. It is important as a staple crop in many areas around the world (e.g., highlands of Asia and Africa), but is also gaining popularity for human consumption in western diets due to its high nutritional value (Meints et al. 2016). Due to the large scientific community researching barley, it is a suitable model crop for studying the implications of climate change and understanding key relationships between science and agriculture (Muñoz-Amatriaín et al. 2014b). Given the scenario of changing environmental conditions affecting key crop production areas, research on biotic and abiotic stresses has become more critical. Two of the most important diseases affecting barley production are stem rust and stripe rust, caused by Puccinia graminis f. sp. tritici and $P$. striiformis f. sp. hordei, respectively (Dean et al. 2012). These diseases can have a significant negative impact on barley yield and malting quality through reductions in kernel components such as

†Corresponding author: P. M. Hayes; patrick.m.hayes@oregonstate.edu

Funding: Support was provided by Comision Nacional de Investigacion Cientifica y Tecnologica (CONICYT) Becas-Chile 72160554 and the U.S. Department of Agriculture (grants 58-2050-6-005 and 58-2072-5-037).

*The $\boldsymbol{e}$-Xtra logo stands for "electronic extra" and indicates that five supplementary figures and supplementary tables are published online.

The author(s) declare no conflict of interest.

(C) 2020 The American Phytopathological Society plumpness, weight, and germination (Chen and Kang 2017; Roelfs 1985; Steffenson 1992).

P. graminis f. sp. tritici race TTKSK (isolate Ug99) and its variants are a serious threat to wheat and barley production worldwide (Singh et al. 2015; Steffenson et al. 2017). Cereal rust pathogens, such as race TTKSK, are a persistent problem for cereal production as spores can migrate within and across continents and become adapted to prevailing conditions (Ali et al. 2014). The most common stem rust resistance gene in barley $(R p g l)$ is not effective against TTKSK; thus, barley production is vulnerable if this race is introduced to the region (Steffenson et al. 2013, 2017). The rpg4/ Rpg5 complex is the only locus known to confer resistance to TTKSK in barley (Steffenson et al. 2009; Zhou et al. 2014). This complex contains three tightly linked genes (Rpg5, HvRgal, and $H v A d f 3$ ) that are inherited as a single unit. Markers targeting polymorphisms in Rpg 5 have been used to introgress this complex into different elite barley lines (Derevnina et al. 2014; Sharma Poudel et al. 2018). However, this complex appears to interact with other genes when it is introgressed into different genetic backgrounds (Hernandez et al. 2019). More detailed studies are needed to evaluate the introgression of rpg4/Rpg 5 into a wide range of genetic backgrounds.

Over the past 40 years, the barley stripe rust (BSR) pathogen has moved around the world. It is most problematic in production areas with cool wet weather, where it can cause yield losses of up to $70 \%$ (Chen and Kang 2017; Chen et al. 1995; Line 2002). Since its initial detection in Colombia in 1975 (Dubin and Stubbs 1986), the disease spread throughout the Americas, reaching the United States in 1991. Since then, the disease has become a constant threat to barley production (Chen et al. 1995; Marshall and Sutton 1995; Roelfs et al. 1992). Breeders can deploy genetic resistance using major effect resistance genes of which at least 26 different ones are known 
(Chen and Line 1999, 2003). Additionally, numerous other studies have identified and mapped quantitative trait loci (QTL) for resistance at the seedling and adult plant stages (Belcher et al. 2018; Castro et al. 2003a; Esvelt Klos et al. 2016; Gutiérrez et al. 2015).

Although fungicides can protect barley against these rusts and other fungal pathogens, host resistance is the preferred method of control (Chen and Kang 2017), as growing resistant cultivars is more economically and environmentally appropriate (Steffenson and Smith 2006). Two major types of resistance have been described and targeted. All-stage resistance is expressed at all growth stages, usually controlled by genes conferring major effect qualitative resistance (gene-for-gene), and is primarily race specific. By contrast, adult plant resistance is expressed only at the adult growth stage, is manifested by a slow progression of disease development that is quantitatively inherited, and is not markedly affected by different pathogen races (Castro et al. 2003b; Chen and Kang 2017; Mundt 2018). Although introgression of qualitative resistance genes is relatively simple, the risks involved in deploying gene-for-gene resistance are well known as new virulent races can more easily overcome resistance (Chen 2005; Parlevliet 1983). Adult plant resistance has greater potential to be durable, but multigene inheritance requires extensive field testing. One successful approach is to develop lines that carry both all-stage resistance and adult plant resistance in order to provide more durable resistance. However, if the resistance genes being introgressed are from exotic sources, it can be a challenge to maintain regional adaptability and agronomic performance (Steffenson and Smith 2006). Current genetic tools, such as marker assisted selection, can enable plant breeders to map and introgress desirable rust resistance genes and QTLs into adapted barley germplasm more efficiently and productively (Esvelt Klos et al. 2016; Yan and Chen 2006). The molecular marker-facilitated incorporation of multiple resistance genes into a single barley genotype (gene pyramiding) has proven to be an effective way to achieve durable resistance for several plant pathogens (Mundt 2018).

Access to germplasm resources, collaborative phenotyping, open-source tools for QTL analysis, and more affordable DNA sequencing/genotyping have had a synergistic effect in terms of facilitating progress in identifying alternative alleles at known disease resistance loci and QTLs. A key tool for genetics research is the reference barley genome sequence (Mascher et al. 2017) because it facilitates the integration of phenotypic data with different sequencing platforms to identify candidate genes (MuñozAmatriaín et al. 2014b). Thousands of markers can be used to scan the entire genome for QTLs and to subsequently fine-map their determinants (Bayer et al. 2017; Comadran et al. 2012). Highthroughput SNP genotyping platforms have been widely used to genotype populations and/or multiple accessions for genetic diversity analysis (Muñoz-Amatriaín et al. 2014a), association analysis (Belcher et al. 2018; Case et al. 2018b), and QTL mapping (Esvelt Klos et al. 2016; Sharma Poudel et al. 2018).

The aim of this study was to evaluate the introgression of the rpg4/Rpg5 resistance gene complex in a diverse set of barley lines with varying degrees of stripe rust resistance. Accordingly, the objectives of this paper were to (i) identify the effects of the rpg4/ Rpg 5 complex introgression at the seedling and adult plant stages in a set of lines with different genetic backgrounds; (ii) map other determinants of resistance interacting with the rpg4/Rpg5 complex at both stages; (iii) map genomic regions associated with resistance to stripe rust in the same population; and (iv) identify lines carrying resistance to both stem rust and stripe rust as potential breeding material. This research benefited from the framework of prior reports on genes and QTLs conferring resistance to stem rust (Case et al. 2018b; Hernandez et al. 2019; Sallam et al. 2017; Sharma Poudel et al. 2018) and stripe rust (Castro et al. 2002b, 2003b; Esvelt Klos et al. 2016; Richardson et al. 2006; Vales et al. 2005).

\section{MATERIALS AND METHODS}

Plant materials. A set of 358 doubled haploid barley lines (hereafter referred to as Cycle II) were derived from eight crosses among five TTKSK-seedling resistant selections from Cycle I (Hernandez et al. 2019), five putatively TTKSK-seedling susceptible Oregon State University (OSU) breeding lines, and three potentially TTKSK-seedling resistant sources from University of California (UC)-Davis (Table 1). The selection of Cycle I parents was based on their phenotype as well as the presence of the resistance allele Rpg 5 and the most significant SNPs defining QTLs on $5 \mathrm{H}$ and $7 \mathrm{H}$ as reported by Hernandez et al. (2019). The susceptible parents were selected based on other positive attributes, such as malting quality, naked caryopsis, and resistance to stripe rust, leaf rust and/or leaf scald. Based on data from stem rust nurseries grown at Njoro, Kenya and Debre Zeit, Ethiopia from 2010 to 2017, UC-Davis lines (UC1231L, UC1322, and UC1266) were moderately susceptible to moderately resistant to prevailing races of stem rust at the adult plant stage. Of the 358 progeny, 281 are two-rowed, 77 are six-rowed, 31 require vernalization, 327 do not require vernalization, 305 are covered (hulled), and 53 are naked (hulless).

Resistance to stripe rust at the adult plant stage. BSR field evaluations were conducted in 2018 and 2019 at Corvallis, OR (denoted CV18 and CV19) and Davis, CA (DV18 and DV19). A combination of location-years was used to evaluate adult plant resistance for BSR. Lines were planted in 1-m single rows with $20 \mathrm{~cm}$ between rows. For the 2018 and 2019 seasons, trials were planted 27 and 17 October in Corvallis and 14 and 12 November in Davis, respectively. Randomized complete block designs with two replicates and three common checks were used in all environments. The checks were as follows: (i) Robust, a highly susceptible sixrowed spring barley; (ii) Thoroughbred, a moderately to highly susceptible six-rowed winter variety; and (iii) DH130910, a tworowed facultative selection from the OSU barley breeding program with resistance to several diseases, including BSR. Due to seed availability problems, checks DH130910 and Thoroughbred were replaced with Full Pint (resistant) and Baronesse (susceptible) in DV18. Both are two-rowed springs. A border composed of an equal mixture of Robust and Thoroughbred surrounded each experiment. Artificial inoculation was conducted to augment natural infection in all trials, except for CV18. At Davis, rust inoculations were carried out on the susceptible spreader plants (at Zadoks growth stage [GS] 31 to 34) (Zadoks et al. 1974) by needle-injecting into stems a mixtures of urediniospores of three BSR races ( $P$. striiformis $\mathrm{f}$. sp. hordei 46, $P$. striiformis f. sp. hordei 48 , and $P$. striiformis $\mathrm{f}$. sp. hordei 72) collected in the same field the previous season. At Corvallis, artificial inoculation was performed in 2019 using BSR races $P$. striiformis f. sp. hordei 33 and $P$. striiformis $\mathrm{f}$. sp. hordei 52, kindly provided by Xianming Chen (USDA-ARS) and previously increased in a plant growth chamber. A mixture of the susceptible checks (Robust and Thoroughbred) was used as a spreader and planted every five rows throughout the experiment. The spreaders were inoculated with a mixture of spores and talcum powder (in a 1: 10 ratio) twice, 10 days apart, during the tillering stage (Zadoks GS 29 to 31). In CV19 and DV18, adult plant reactions were evaluated three times between the heading (Zadoks GS 50) and grain filling stages (Zadoks GS 80), when the susceptible checks exhibited an infection level above 50\% severity. In DV19 and CV18, a single rating was taken on each plot around Zadoks GS 80. Disease severity was rated as the percentage of diseased leaf area on a singlerow plot basis. For those environments with multiple ratings throughout the season, the highest score for each line was used for association mapping. A scale from 0 to 9 was used for recording the infection types (ITs) where $0=$ no visible signs or symptoms and $9=$ abundant sporulation, with no necrosis or chlorosis (McNeal et al. 1971). Heading date was recorded as the Julian calendar day when $50 \%$ of heads in a plot emerged at both locations in both years. 
Resistance to stem rust at the seedling stage. The Cycle II panel, parents, and checks were scored for their ITs to $P$. graminis $\mathrm{f}$. sp. tritici TTKSK in the Biosafety Level 3 Containment Facility at the University of Minnesota (St. Paul, MN). A randomized complete block design with two replicates and replicated controls was used. The accessions Q21861 (PI 584766) and Q/SM20 were used as resistant controls. Q21861 carries Rpg1 and rpg4/Rpg5 resistance gene complex, whereas Q/SM20 carries only the rpg4/ Rpg5 gene complex derived from Q21861. Hiproly (PI 60693) and PI 532013 were used as susceptible controls; these accessions have susceptibility alleles at both loci. When the first leaves of plants were fully expanded (five plants per pot), they were inoculated with P. graminis f. sp. tritici TTKSK and evaluated for their ITs using a 0 to 4 scale 12 to 14 days postinoculation as described in Steffenson et al. (2017). Briefly, seeds were planted in a greenhouse and grown at 20 to $22^{\circ} \mathrm{C}$ with a 14 to $16 \mathrm{~h}$ photoperiod supplemented by $400-\mathrm{W}$ sodium vapor lamps. Rust isolate $04 \mathrm{KEN} 156 / 04$ of race TTKSK (previously increased on susceptible wheat host McNair 701) was used as the inoculum source. For inoculation, a urediniospore suspension $(0.017 \mathrm{mg}$ of uredinospores per $1 \mathrm{ml}$ of oil) was applied at a rate of $0.149 \mathrm{mg}$ of rust per plant. Inoculated plants were then moved to a dark mist chamber where the relative humidity was maintained near $100 \%$. After 12 to 14 days, stem rust ITs were assessed based on the 0 to 4 scale developed by Stakman et al. (1962) as modified for barley by Miller and Lambert (1955). For association analysis, IT scores were transformed to a categorical value as described by Zhou et al. (2014). A coefficient of infection (CI) threshold of 2.7 was used to classify germplasm as resistant (CI $\leq 2.7)$ or susceptible $(\mathrm{CI}>2.7)$ based on previous reports (Hernandez et al. 2019; Sallam et al. 2017).

Resistance to stem rust at the adult plant stage. The Cycle II panel was evaluated for stem rust resistance at the adult plant stage in field trials conducted at the University of Minnesota (St. Paul, MN) during two growing seasons (MN18 and MN19). Due to the harsh winters in Minnesota, the trial was spring planted (7 May 2018 and 14 May 2019), and only barley lines without a vernalization requirement (spring and potentially facultative types) were successfully evaluated. Heading dates were recorded as days after planting in both years. Out of 358 lines, 327 exhibited complete stem elongation during both growing seasons (i.e., they did not require vernalization) and were used for association analysis. Disease assessment was performed when plants were between the heading and hard dough stages of development (Zadoks GS 55 to 87). P. graminis f. sp. tritici race QCCJB was used to evaluate resistance at the adult plant stage in this nursery. We used this race as a surrogate for race TTKSK due to the impossibility of using race TTKSK for field trials in the United States. The resistance conferred by rpg 4/Rpg 5 complex to both races has been shown to be genetically equivalent (Arora et al. 2013; Steffenson et al. 2009; Wang et al. 2013). Q21861, Steptoe, and PI 532013 were used as checks. Q21861 is the resistant check, possessing both Rpg 1 and the rpg4/Rpg5 resistance complex. Steptoe and PI 532013 are susceptible and highly susceptible controls, respectively. A randomized complete block design with two replications was used in both years. Strips of paired plots were surrounded by two continuous rows of a susceptible barley spreader; this facilitated the even distribution of inoculum across the experiment. The spreaders were needle-injected with $P$. graminis f. sp. tritici QCCJB urediniospores at the tillering stage (Zadoks GS 29 to 31) as described by Case et al. (2018b). Briefly, $1 \mathrm{~g}$ of fresh urediniospores was suspended in 1 liter of distilled water and 6 drops of $20 \%$ Tween 20 . Then, $1 \mathrm{ml}$ of solution was injected into the stems of spreader plants a 1 meter intervals. A direct foliar inoculation was also done to augment disease levels in the nursery. For this inoculation, a hand-held ultra-low sprayer (Mini-ULVA, Micron Group, Herefordshire, UK), was used to apply the spores at a rate of 1 liter per 800 plots. Resistance and the adult plant stage was assessed visually as disease severity ( 0 to $100 \%$ ) on stem and leaf sheaths of each tested line using a modified Cobb scale (Peterson et al. 1948).

Phenotypic data analysis. Histograms were used to visualize the phenotypic data distributions, including checks, obtained from each environment. To improve normality of the original phenotypic data, the mean of each line was subjected to $\log _{10}$, square root, and arcsine transformations. The Shapiro-Wilk normality test was performed in R (R Development Core Team 2015) to compare the normality of transformed and untransformed data. Pearson correlation coefficients for severity between diseases and across environments were calculated in R to determine the consistency of disease assessments. Variances for genotype, environments, and genotype by environment interactions were estimated in a mixed

TABLE 1. Parental accession information of the Cycle II barley population

\begin{tabular}{|c|c|c|c|c|c|c|c|}
\hline Line & Pedigree & Origin & $\mathrm{IT}^{\mathrm{M}} \mathrm{M}^{\mathrm{a}}(\mathrm{GR})^{\mathrm{b}}$ & $\mathrm{CI}^{\mathrm{c}}$ & $\mathrm{MN} 18^{\mathrm{d}}$ & MN19 & Genotype $^{\mathrm{e}}$ \\
\hline DH140512 & SH98076/Full Pint & Oregon, U.S.A. (Oregon State University) & $0 ;(\mathrm{R})$ & 0.5 & 3.0 & 67.5 & rpg4/Rpg5 \\
\hline DH140278 & SH98076/Full Pint & Oregon, U.S.A. (Oregon State University) & $00 ;(\mathrm{R})$ & 0.1 & 5.0 & 41.0 & rpg4/Rpg 5 \\
\hline DH140279 & MC0181-11/Full Pint & Oregon, U.S.A. (Oregon State University) & $0 ; 1-(\mathrm{R})$ & 0.8 & 6.5 & 80.0 & rpg4/Rpg5 \\
\hline DH140030g & SH98076/10.1151 & Oregon, U.S.A. (Oregon State University) & $0 ;(\mathrm{R})$ & 0.5 & NA & NA & rpg4/Rpg5 \\
\hline DH140213 & SH98076/10.1151 & Oregon, U.S.A. (Oregon State University) & $1-0 ;(\mathrm{R})$ & 1.3 & 3 & 6 & rpg4/Rpg5 \\
\hline Thunderg & Wintmalt/Charles & Oregon, U.S.A. (Oregon State University) & $0 ; 1-(\mathrm{R})$ & 0.8 & NA & NA & rpg4/Rpg5 \\
\hline $10.0860^{\mathrm{g}}$ & Wintmalt/Charles & Oregon, U.S.A. (Oregon State University) & 23-1 (MS) & 3.1 & NA & NA & rpg4/Rpg5 \\
\hline$U C 1231 L$ & F6 22IBYT7//UC933/UC1047 & California, U.S.A. (UC-Davis) & $3-2(\mathrm{~S})$ & 3.4 & 7.5 & 17.5 & _ \\
\hline UC1322 & Z05500120/CIMMYT 7862 & California, U.S.A. (UC-Davis) & $0 ;(\mathrm{R})$ & 0.5 & 5.0 & 27.5 & - \\
\hline$U C 1266$ & Tamalpais (UC1134)//Madera/UC937 & California, U.S.A. (UC-Davis) & $10 ;(\mathrm{R})$ & 1.6 & 7.5 & 15.0 & - \\
\hline DH130939 & Full Pint/VIOLETTA & Oregon, U.S.A. (Oregon State University) & $0 ; 1(\mathrm{R})$ & 0.9 & 17.5 & $\mathrm{NA}^{\mathrm{h}}$ & - \\
\hline DH130004 & $\begin{array}{l}\text { SHORT11-7 (TC6W265)//HERZ 29494/ } \\
2991 \text { (35) }\end{array}$ & Oregon, U.S.A. (Oregon State University) & 23-1 (MS) & 3.1 & 25.0 & $\mathrm{NA}^{\mathrm{h}}$ & - \\
\hline DH120412g & $\begin{array}{l}\text { SHORT11-7 (TC6W265)//HERZ 29494/ } \\
2991 \text { (35) }\end{array}$ & Oregon, U.S.A. (Oregon State University) & $10 ;(\mathrm{R})$ & 1.6 & NA & NA & - \\
\hline
\end{tabular}

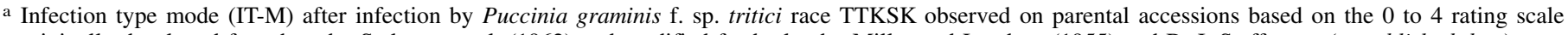
originally developed for wheat by Stakman et al. (1962) and modified for barley by Miller and Lambert (1955) and B. J. Steffenson (unpublished data).

b General reaction (GR): MR, moderately resistant; MS, moderately susceptible; R, resistant; and S, susceptible.

c Coefficient of infection (CI) mean from two replicates calculated as described by Zhou et al. (2014).

d Disease severity after infection by $P$. graminis f. sp. tritici race QCCJB under field conditions in Minnesota during 2018 and 2019 (MN18, MN19).

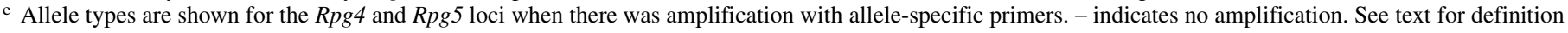
of $\operatorname{Rpg} 5 X x$.

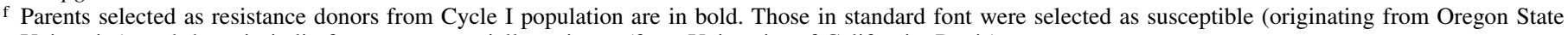
University), and those in italic font were potentially resistant (from University of California, Davis).

g Parents with vernalization requirements were not evaluated under spring planting conditions.

h Seed not available for these parents during MN19. 
linear model using lme4 in R, where variables were included as random effects. Broad-sense heritability $\left(H^{2}\right)$ estimates were calculated for each disease using the restricted maximum likelihood method (REML) by a random effect model. The best linear unbiased predictions (BLUPs) for each line across environment were obtained.

Genotyping. Leaf tissue from the Cycle II panel was collected at the seedling stage from single plants grown in a greenhouse at OSU. Leaf tissue was sent to the USDA-ARS Regional Small Grains Genotyping Laboratory at Fargo, ND for DNA extraction and genotyping. The panel was genotyped using the barley $50 \mathrm{~K}$ Illumina iSelect SNP array (Bayer et al. 2017), and allele calls were made using Genome Studio software (Illumina, San Diego, CA). The single nucleotide polymorphisms (SNP) markers were filtered to remove markers with a minor allele frequency of less than $5 \%$, missing values at greater than $10 \%$, and heterozygosity at greater than $5 \%$. This filtering process resulted in a set of 28,587 markers. Missing genotyping information was imputed using a stochastic imputation method based on allele frequency using an R custom script (R Development Core Team 2015) Additional SNP information, such as chromosome and base pair position, was obtained from two online resources, BARLEYMAP (Cantalapiedra et al. 2015) and the IPK Barley BARLEX server (https:// webblast.ipk-gatersleben.de/) based on the reference genome (Mascher et al. 2017).

Linkage disequilibrium, population structure, and kinship characterization. Markers with known map coordinates were analyzed with TASSEL v5 (Bradbury et al. 2007) to calculate the linkage disequilibrium (LD) squared allele frequency correlation $\left(r^{2}\right)$ estimates using a sliding window of 25 adjacent markers to identify nonredundant SNPs. Out of 28,587 SNPs, 9,918 markers were in perfect $L D$ with another adjacent marker $(L D=1)$ and were removed, resulting in a final number of 18,669 SNP markers. This final number of markers was used for the calculation of a kinship matrix and subsequently for the genome-wide association study (GWAS). Principal component analysis was performed in R (R Development Core Team 2015) using 18,669 markers, and the output was used as a covariate in the association analysis.

GWAS. Phenotypic data obtained for stripe and stem rust for each line and the filtered set of 18,669 SNP markers were used to identify marker-trait associations for resistance to both diseases using TASSEL v5 software (Bradbury et al. 2007). Marker-trait associations for resistance at the adult plant stage were evaluated for all single environments and BLUPs across environments. Association mapping was conducted using three different models: (i) a general linear model accounting only for population structure $(Q$ model), (ii) a mixed linear model accounting only for kinship ( $K$ model), and (iii) a mixed linear model including population structure as a fixed effect and kinship as a random effect $(Q+K$ model). The $Q$ model can be defined as $y=X a+Q v+e$; the $K$ model can be denoted as $y=X a+Z u+e$; and the $Q+K$ model can be defined as $y=X a+Q v+Z u+e$, where $y=$ the vector of mean severity for each line, $a=$ the vector of marker effects, $v=$ the vector of fixed population effects, $u=$ random genetic background effects, and $e=$ residuals. $X, Q$, and $Z$ are incidence matrices that connect response variables with effects. To account for population structure, the first three principal components from the PCA were included. A kinship matrix was estimated using the final SNP set $(18,669$ markers) to account for genetic relatedness among lines in the Cycle II panel. To avoid false positive associations arising from multiple test comparisons in the evaluated models, a false discovery rate $(q<$ 0.1 ) was used to control for Type I error in R (Benjamini and Hochberg 1995). SNPs that were associated with resistance and landed in the same genomic region were assumed to detect the same QTL if the LD between them was greater than 0.5 and they showed consistent direction effects. The marker with the strongest association was used as a tag SNP. The effects of different allele combinations was performed using lines carrying Rgp5 and loci that were significantly associated with stem rust resistance at both growth stages. The most significant marker at each locus was used to identify lines carrying specific genotypes. For the adult plant stage, the same marker was used for allele combination analysis in both years. To further compare mean differences among different allele combinations, a Tukey's comparison test $(\alpha=0.05)$ was performed.

Candidate genes. Using the BARLEYMAP database (Cantalapiedra et al. 2015), common markers were used to compare whether the QTLs detected in this study were aligned with previously reported QTLs. As reported by Sallam et al. (2017), a $2-\mathrm{Mb}$ region surrounding the most significant marker at a defined QTL peak was used to search for high confidence genes in the reference genome assembly (Mascher et al. 2017).

\section{RESULTS}

Phenotypic analysis. Stripe rust resistance. The Cycle II panel was tested in four environments (CV18-19, DV18-19) for resistance to stripe rust at the adult plant stage. Controls reacted as expected. DH130910, the resistant control, exhibited low to moderate severity scores across all environments, ranging from 1.5 to $20 \%$. Robust and Thoroughbred, both used as susceptible checks, consistently showed higher severity values, ranging from 35 to $85 \%$ and 20 to $80 \%$, respectively (Supplementary Fig. S1). As data transformation did not improve normality, raw data were used for all analyses. Significant differences were observed among lines, with phenotypic means ranging from 9 to $36 \%$ across environments and an overall mean of $21 \%$. Genetic variance, error variance, and broad-sense heritability were estimated across all environments. Variance components for genotypes were highly significant at both locations, and environment and $\mathrm{G} \times \mathrm{E}$ effects were also significant. Heritabilities were moderate to high, ranging from 0.62 in Corvallis to 0.74 in Davis. The IT rating exhibited the highest heritability at 0.83 (Supplementary Table S1). Because a high correlation between disease severity and IT was observed for both years in Davis $(r=$ 0.80 ), only severity values will be reported hereafter. Pearson correlations for BSR severity within and across locations were 0.71 and 0.68 , respectively (Supplementary Table S3). According to the BLUP analysis across locations, 97 lines (26.2\%) were highly resistant (mean SEV $=4.4 \%$ ) compared with the 68 (18.3\%) highly susceptible lines (mean SEV $=48.4 \%$ ) (Fig. 1B).

Stem rust resistance at seedling stage. As shown in Figure 1A, at the seedling stage the resistant and susceptible checks behaved as expected. Some of the parents and progeny, however, did not behave as expected. As shown in Table 1, the Cycle I resistant parents all had resistant phenotypes. However, three of the OSU "susceptible" parents (Thunder, DH130939, and DH120412) chosen as recipients for introgression of the rpg4/Rpg 5 complex were actually resistant. Two of three putatively resistant UC-Davis parents (UC1322 and UC1266) were also resistant. The resistance of these parents was confirmed in a second round of phenotyping (Supplementary Table S2). The resistance status of the OSU "susceptible" parents was further confirmed by the resistant phenotypes of their progeny (Table 2). Segregation for resistance was observed for just one of the three parents but in a low percentage ( 5 out of 67 lines susceptible), and one of the two resistant UC-Davis selections segregated for resistance. Using a resistance threshold of $\mathrm{CI} \leq 2.7,88 \%(n=311)$ of the progeny were resistant to TTKSK and only $11 \%(n=40)$ were susceptible. Within the resistant class, $75 \%$ of the progeny had CI values less than or equal to the resistant controls (Q21861 and Q/ SM20).

Resistance to stem rust at the adult stage. The adult plant stage disease severities were markedly different from the seedling stage disease evaluations and they were also different across years (MN18 versus MN19). Parental lines and progeny with vernalization requirements could not be assessed under spring planted conditions. Therefore, data on these entries are missing in Tables 1 and 2. The 
adult plant data are thus based on $327 \mathrm{DH}$ lines, nine parents, and three checks. Disease severities were higher in 2019 than in 2018 as evidenced by Steptoe (23\% in MN18 and 60\% in MN19) and PI 532013 (40\% in MN18 and 50\% in MN19. The resistant check, Q21861, also had a higher disease severity in 2019: 3\% (MN18) versus 22\% (MN19). The higher disease severities in 2019 may be due to maximum daily temperatures over $27^{\circ} \mathrm{C}$ in a 6-day period during the logarithm phase of rust epidemic. These temperatures likely reduced the effectiveness of the rpg4/Rpg 5 complex, which is sensitive to high temperatures (Jin et al. 1994; Steffenson et al. 2017). Higher inoculum pressure may also have played a minor role.

The progeny also showed different phenotypic distributions for resistance at the adult plant versus seedling stage, and between years for the adult plant reaction (Table 2, Supplementary Fig. S2). At the seedling stage, there were markedly more resistant lines compared with adult plant stage evaluations in MN18 and MN19 (Fig. 2). Using the mean of check Q21861 plus one standard deviation as a threshold to identify resistant lines within each year, 40 (12\%) of the progeny were resistant in 2018 (threshold $=5.3 \%$ ), whereas in 2019 (threshold $=33 \%), 141(43 \%)$ of the progeny had lower levels of disease than Q21861. However, possibly due to higher temperatures in 2019, the number of resistant progeny may be inflated by partial escapes due to early maturity. Therefore, the 2019 data were divided into two groups: early heading date $(\mathrm{HD}<59, n=168)$ and late
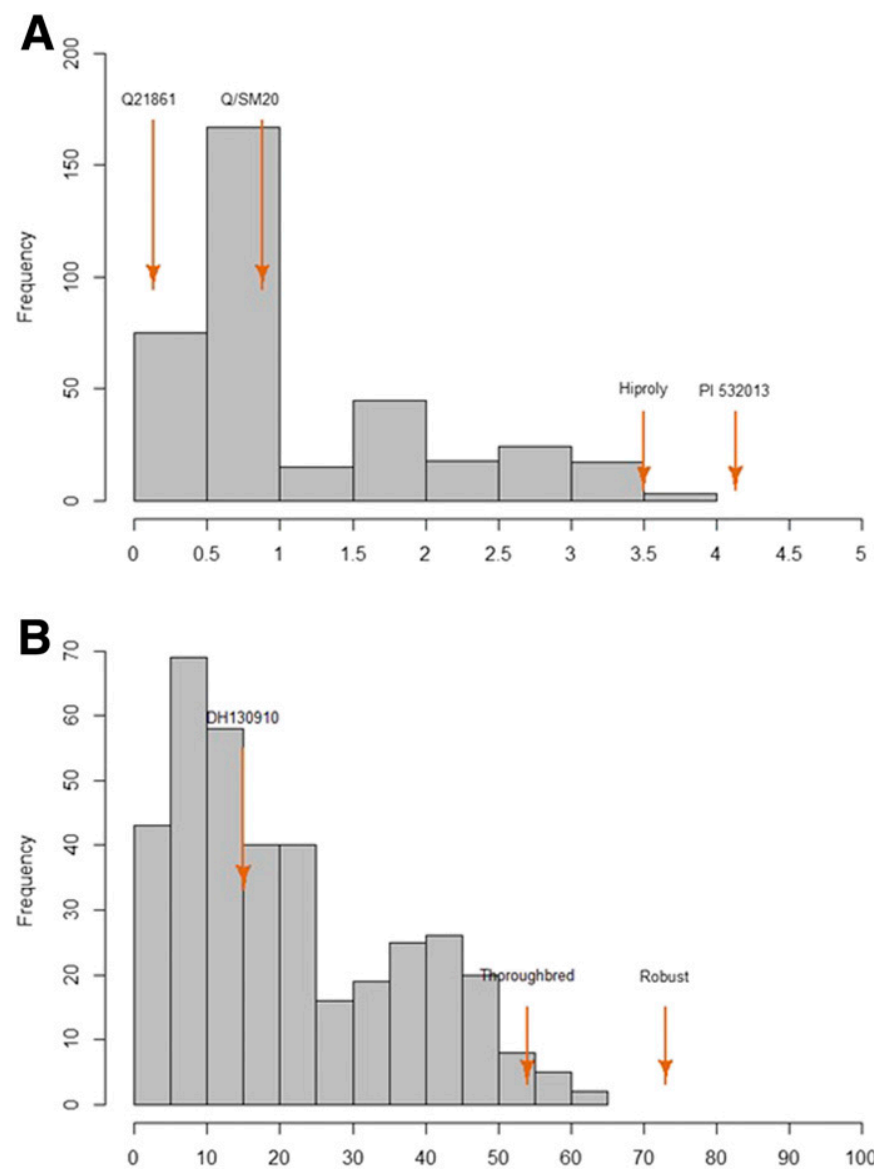

Fig. 1. Phenotypic frequency distribution for $\mathbf{A}$, seedling resistance, expressed as coefficient of infection (CI) values, after infection by Puccinia graminis $\mathrm{f}$. sp. tritici race TTKSK and $\mathbf{B}$, best linear unbiased predictions (BLUPs) across four environments (Corvallis 2018-2019 and Davis 2018-2019) for disease severity at the adult plant stage, expressed as percentage (\%) in response to Puccinia striiformis f. sp. hordei in the Cycle II barley population. CI and severity mean values for resistant and susceptible checks are shown. The phenotyping for seedling resistance to stem rust was conducted in a Biosafety Level 3 Containment Facility at the University of Minnesota. heading date (HD $\geq 59, n=157$ [MN19HD59]) (Supplementary Fig. S3). In the early HD group, $98(58 \%)$ lines were classified as resistant based on comparison with the check, whereas only 43 (27\%) lines were classified as resistant in the late HD group (MN19HD59). Using a predefined severity threshold of $10 \%$ in order to adjust for the performance of resistant check across years, fewer resistant lines in MN19HD59 than in MN18 were observed (Fig. 2). As shown in Supplementary Figure S4, there were always fewer resistant lines at the adult plant stage compared with the seedling stage, but the number of resistant lines at the adult stage is a function of comparing to a control or a defined threshold.

Allele-specific genotyping for Rpg5. All of the Cycle I TTKSK resistant parents were positive for Rpg5 (the dominant resistance allele) (Table 1). Of the putatively susceptible OSU parents, none were positive for Rpg5. Two (Thunder and 10.0860) were positive for rpg5 (the recessive susceptibility allele). Three (DH130939, DH130004, and DH120412) showed no amplification product. Genotypes that failed to amplify for either allele at the Rpg 5 locus, and that were resistant at the seedling stage, are referred to as $R p g 5 X x$ in the remainder of this report. At the seedling stage, one of the two of the $r p g 5$ allele types was resistant and two of the Rpg5Xx types were resistant. The three UC-Davis putatively resistant parents all showed no amplification product (Rpg5Xx). At seedling stage, two of the three UC-Davis lines with Rpg5Xx exhibited a resistance reaction (Table 1 ).

Of the 358 progeny in Cycle II, 190 (53\%) were positive for Rpg5, 29 (8\%) were positive for $r p g 5$, and 139 (39\%) were Rpg5Xx. As shown in Table 2, progeny segregated as expected for allele type at the Rpg 5 locus. For example, progeny of Rpg $5 \times R p g 5 X x$ crosses segregated $1: 1$. Using a threshold of $C I \leq 2.7$ to define resistance, at the seedling stage 177 of the Rpg5 progeny were resistant and 10 were susceptible; 21 of the rpg 5 progeny were resistant and 8 were susceptible. Of the Rpg5Xx types, 25 were susceptible, and 113 were resistant, with 9 of these rated as highly resistant (mode 00; $\mathrm{CI}=0.125$ ). In terms of resistance at the adult plant stage, where only parents and progeny without a vernalization requirement could be evaluated, $9.4 \%$ of lines carrying Rpg 5 expressed equal or lower disease severity compared with control Q21861 and only 3.3\% were susceptible compared with PI 532013 during 2018. In 2019, a remarkable increase in the number of susceptible lines carrying $\operatorname{Rpg} 5$ (24.5\%) was observed along with an increase in the number of resistant lines (15.3\%). A similar trend was observed for lines carrying $R p g X x$ in both years (Supplementary Table S4). Considering adult plant disease severity in 2018 and 2019 (MN19HD59), seven (2\%) lines had an average disease severity mean of $\leq 6 \%$ (Supplementary Table S5). Of these, five were positive for Rpg 5 and two were positive Rpg $5 X x$. All were resistant at the seedling stage.

Genome wide association analysis. Stripe rust. The model including PC and K was used for the analysis (PC + K model) and heading date was included as covariate in the model. Four loci, two located on the short arm of $1 \mathrm{H}$, one on $4 \mathrm{H}$, and one on $5 \mathrm{H}$ were associated with resistance to BSR based on BLUPs obtained across all locations and years (Fig. 3E). The QTL peak on $1 \mathrm{H}$ was subdivided into two resistance QTLs: 1 H.a at $3700002 \mathrm{Mb}(4.96$ $\mathrm{cM})$ and 1H.b at $8935735 \mathrm{Mb}(11.46 \mathrm{cM})$ based on linkage disequilibrium analysis $\left(R^{2}<0.2\right)$ using the most significant markers at each locus, JHI-Hv50k-2016-3887 and BOPA2_12_ 30918 , respectively. The most significant marker on $4 \mathrm{H}$ was JHIHv50k-2016-264205 at $616425085 \mathrm{Mb}$ (97.45 cM). Marker JHIHv50k-2016-311988 at $529074874 \mathrm{Mb}(72.8 \mathrm{cM})$ was the most significant SNP on $5 \mathrm{H}$. Total phenotypic variation for stripe rust explained by those four QTL reached a maximum of $46 \%$ (Table 3 ).

Stem rust resistance at seedling and adult stages. At the seedling stage, two QTLs were found to be associated with resistance to stem rust. As shown in Figure 3A and Table 3, the largest effect is on chromosome $5 \mathrm{H}$ and is located at the genome coordinates of Rpg5. The second QTL is on chromosome $3 \mathrm{H}$ 
(QTL3). The adult plant phenotype data from MN18 revealed two QTLs: both on 5H (Fig. 3B and Table 3). One of the QTLs is at the genome coordinates of Rpg 5 and the other is at $70.14 \mathrm{cM}$. Using all phenotype data from MN19, one QTL was detected on chromosome 2H (Fig. 3C and Table 3). This QTL coincides with the genome coordinates of Ppd-H1, a flowering time locus (Digel et al. 2016). When heading date was included as a cofactor with the complete data set, no QTL were detected. GWAS was then performed on the two subgroups of the 2019 data (HD $<59$ and HD $\geq 59$ ). In the early group, no QTLs were detected. In the late group, a QTL on 5H was identified (Fig. 3D and Table 3). The most significant SNP at this QTL is $\sim 5.4 \mathrm{cM}$ proximal to the most significant marker for the resistance QTL at the adult plant stage identified in MN18.

Using Rpg 5 allele-specific amplification data, lines with different allele combinations for Rpg5 and QTL3 were compared for stem rust resistance at the seedling stage (Supplementary Fig. S5). Upper and lowercase letters were used to indicate resistance and susceptibility alleles, respectively. Significant mean differences for stem rust severity were observed among haplotypes based on CI scores. The CI mean of lines carrying the susceptible rpg5/qtl3 haplotype was higher $(\mathrm{CI}=2.3$ ) compared with the mean of lines carrying the Rpg5/QTL3 haplotype $(\mathrm{CI}=0.8)$. Using the same approach as for the adult plant stage, there were significant interactions between QTL5 and Rpg5 in MN18 and MN19HD59 (Fig. 4). In MN18, the rpg5/qtl5 genotypes had the highest average severity (31\%). In contrast, Rpg5/QTL5 genotypes had an average severity of $13 \%$. Genotypes with the resistance alleles at one locus (e.g., Rpg5/qtl5 and rpg5/QTL5) were not statistically different (24 and 20\%, respectively). In MN19HD59, the rpg5/qtl5 genotypes had an average severity of $64 \%$, whereas the Rpg $5 / Q T L 5$ genotypes had an average severity of $38 \%$, and the $r p g 5 / Q T L 5$ genotypes were not significantly different from this group ( $47 \%$ severity). The mean difference between the Rpg5/QTL5 and Rpg5/qtl5 (57\% severity) genotypes was significant.

Candidate genes. Stripe rust. An inspection of a 2-Mb region surrounding the most significant SNPs using BLUPs was performed to detect candidate genes using the most recently published reference genome assembly (Mascher et al. 2017). At 1H.a, the most significant marker JHI-Hv50k-2016-3887 is $\sim 0.04 \mathrm{Mb}$ downstream of HORVU1Hr1G001540, a disease resistance protein RPM1. This gene has been associated with resistance to Pseudomonas syringae in Arabidopsis (Tornero et al. 2002). The marker BOPA2_12_30918 (1H.b) is $\sim 0.1 \mathrm{Mb}$ from HORVU1Hr1G004140, a disease resistance gene based on the reference genome. The high confidence gene HORVU4Hr1G080720, a leucine-rich repeat receptor-like protein kinase family protein, was located $\sim 0.5 \mathrm{Mb}$ downstream from the most significant marker on 4H (JHI-Hv50k-2016-264205). At 5H, marker JHI-Hv50k2016-311988 is located $\sim 0.3 \mathrm{Mb}$ downstream from the basic leucine-zipper 42 gene HORVU5Hr1G070540, a gene reported as a master regulator of many central developmental and physiological processes including plant defense (Alves et al. 2013).

Stem rust. Two QTLs on $3 \mathrm{H}$ and $5 \mathrm{H}$ were found to be associated with resistance for stem rust at the seedling stage. The most significant marker associated with QTL on $3 \mathrm{H}$ was JHI-Hv50k2016-200274, which is located in the same contig as HORVU3Hr1G081050, a receptor kinase 2 gene that has been associated with pathogen recognition in plants (Goff and Ramonell 2007). The most significant marker associated with the QTL on 5H was SCRI_ RS_155322, located at $640002826 \mathrm{Mb}(150.07 \mathrm{cM})$. This marker is $\sim 0.7 \mathrm{Mb}$ upstream from the rgp4/Rpg5 resistance complex $(640765916 \mathrm{Mb})$ (Mascher et al. 2017). The proximity of the marker associated with resistance to the rpg4/Rpg5 complex suggests that we were able to detect the effect of this complex in our Cycle II barley population, as was also found in other previous studies (Case et al. 2018b; Hernandez et al. 2019; Moscou et al. 2011; Steffenson et al. 2009). At the adult stage, two QTLs were associated with resistance in MN18. The most significant SNP was JHI-Hv50k-2016-311904 on 5H at 528355399 Mb (70.14 cM). This marker is $\sim 0.3 \mathrm{Mb}$ from HORVU5Hr1G070360, a protein kinase family gene, which is involved in a diverse array of plant responses including development, growth, hormone perception, and the response to pathogens (Goff and Ramonell 2007). SCRI_RS_

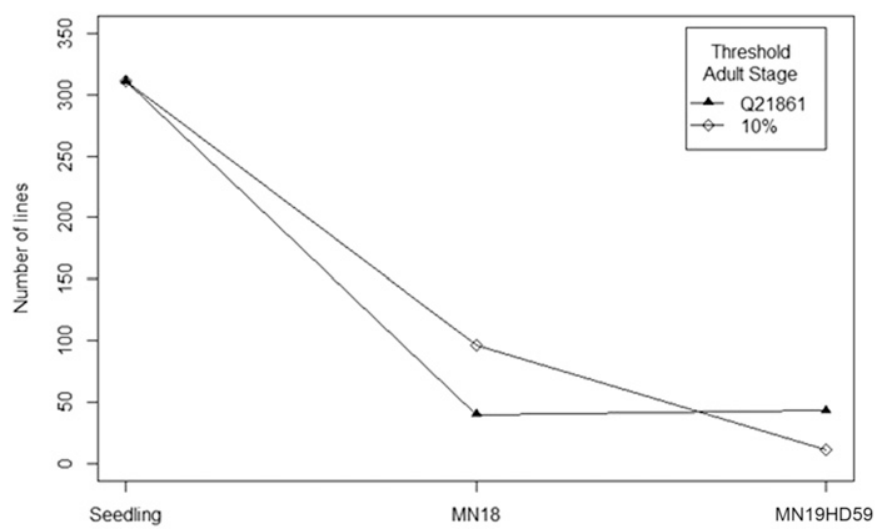

Fig. 2. Number of resistant progeny in the Cycle II population using different thresholds (Q21861 and 10\%) to define resistance, expressed as disease severity after infection by Puccinia graminis f. sp. tritici race QCCJB, at adult plant stage under field conditions at the University of Minnesota in 2018 (MN18) and 2019 (MN19HD59). MN19H59 refers to the subset of lines with heading dates $\geq 59$ days after planting in 2019. At seedling stage, resistance is defined as lines exhibiting a coefficient of infection $\leq 2.7$ after infection by $P$. graminis f. sp. tritici race TTKSK in a Biosafety Level 3 Containment Facility at the University of Minnesota.

TABLE 2. Segregation for resistance to Puccinia graminis f. sp. tritici in Cycle II barley population progeny

\begin{tabular}{|c|c|c|c|c|c|c|c|c|c|c|c|c|c|c|}
\hline \multirow[b]{2}{*}{ Pedigree } & \multicolumn{3}{|c|}{ Seedling $^{\mathrm{a}}$} & \multicolumn{3}{|c|}{ MN18 } & \multicolumn{2}{|c|}{ MN19b } & \multicolumn{3}{|c|}{ MN19HD59c $^{c}$} & \multicolumn{3}{|c|}{ Genotype $^{\mathrm{d}}$} \\
\hline & $\mathrm{N}$ & $\mathrm{R}$ & $\bar{S}$ & $\mathrm{~N}$ & $\mathrm{R}$ & $S$ & $\mathrm{R}$ & $S$ & $\mathrm{~N}$ & $\mathrm{R}$ & $\mathrm{S}$ & $\operatorname{Rpg} 5$ & rpg5 & $\operatorname{Rpg} 5 X x$ \\
\hline DH140512/UC1322 & 26 & 26 & 0 & 26 & 10 & 16 & 15 & 11 & 16 & 9 & 7 & 21 & & 5 \\
\hline DH140512/DH130004 & 75 & 67 & 8 & 71 & 7 & 64 & 32 & 39 & 30 & 9 & 21 & 35 & & 40 \\
\hline DH140512/10.0860 & 44 & 35 & 9 & 33 & 1 & 32 & 7 & 26 & 25 & 5 & 20 & 24 & 19 & \\
\hline DH140278/DH130939 & 46 & 46 & 0 & 46 & 1 & 45 & 9 & 16 & 26 & 3 & 23 & 21 & & 25 \\
\hline DH140278/Thunder & 23 & 23 & 0 & 19 & 0 & 19 & 3 & 37 & 12 & 2 & 10 & 13 & 10 & \\
\hline DH140279/DH120412 & 67 & 62 & 5 & 67 & 2 & 65 & 25 & 42 & 26 & 2 & 24 & 40 & & 27 \\
\hline DH140030/UC1231L & 40 & 27 & 9 & 28 & 5 & 23 & 19 & 8 & 11 & 6 & 5 & 16 & & 24 \\
\hline UC1266/DH140213 & 37 & 25 & 9 & 37 & 14 & 23 & 31 & 5 & 11 & 7 & 4 & 20 & & 17 \\
\hline
\end{tabular}

a $\mathrm{N}=$ total number of progeny per cross. $\mathrm{R}=$ resistant and $\mathrm{S}=$ susceptible as defined as $\mathrm{CI} \leq 2.7$ and $\mathrm{CI}>2.7$, respectively, for seedling reaction after infection by Puccinia graminis f. sp. tritici race TTKSK at the seedling stage.

${ }^{b}$ Adult plant stage, $\mathrm{R}=$ resistant and $\mathrm{S}=$ susceptible as defined as severity $\leq 5.3 \%$ and severity $>5.3 \%$ in MN18 and as $\leq 33.0 \%$ and $>33.0 \%$ in MN19, respectively, after infection by $P$. graminis f. sp. tritici race QCCJB in 2018 and 2019.

c MN19HD59 refers to DH progeny in 2019 with heading dates $\geq 59$ days after planting.

d Allele type at the Rpg5 locus is shown when there was allele-specific amplification and as Rpg5Xx when there was no amplification with allele-specific primers. 
10924 was the most significant marker for the second QTL, which mapped on $5 \mathrm{H}$ at $641259883 \mathrm{Mb}(151.25 \mathrm{cM})$ and corresponds to the rpg4/Rpg5 resistance complex. In MN19HD59, JHI-Hv50k2016-312045 was the most significant marker and is located at $529392430 \mathrm{Mb}(75.56 \mathrm{cM})$. The closest high confidence gene to this marker was HORVU5Hr1G070610.2 (529545296 Mb), a receptor kinase 2 gene.

\section{DISCUSSION}

The results of this experiment highlight key themes in plant disease resistance, breeding, and genetics. These include (i) the importance of developing a catalog of multiple, mapped resistance genes and QTLs, (ii) expression of seedling stage versus adult plant stage resistance, (iii) the influence of environmental factors in disease resistance phenotyping, and (iv) the development of germplasm with resistance to multiple diseases.

New sources of stem rust resistance are an essential component of a long-term disease management strategy. Unfortunately, new sources of resistance are relatively rare, despite extensive screening and characterization efforts (Arora et al. 2013; Case et al. 2018b; Sallam et al. 2017; Steffenson et al. 2017; Zhou et al. 2014). Based on a previous population (Cycle I) generated from a set of different crosses (Hernandez et al. 2019), a new population (Cycle II) was developed to continue studying the implications of introgressing the rpg4/Rpg5 stem rust resistance complex, and QTL alleles discovered in Cycle I, into diverse barley germplasm. Five lines from Cycle I that exhibited high levels of resistance at the seedling stage and had all three resistance alleles (Rpg5, QTL5, and QTL7), were chosen as the resistant parents. The recipients of the
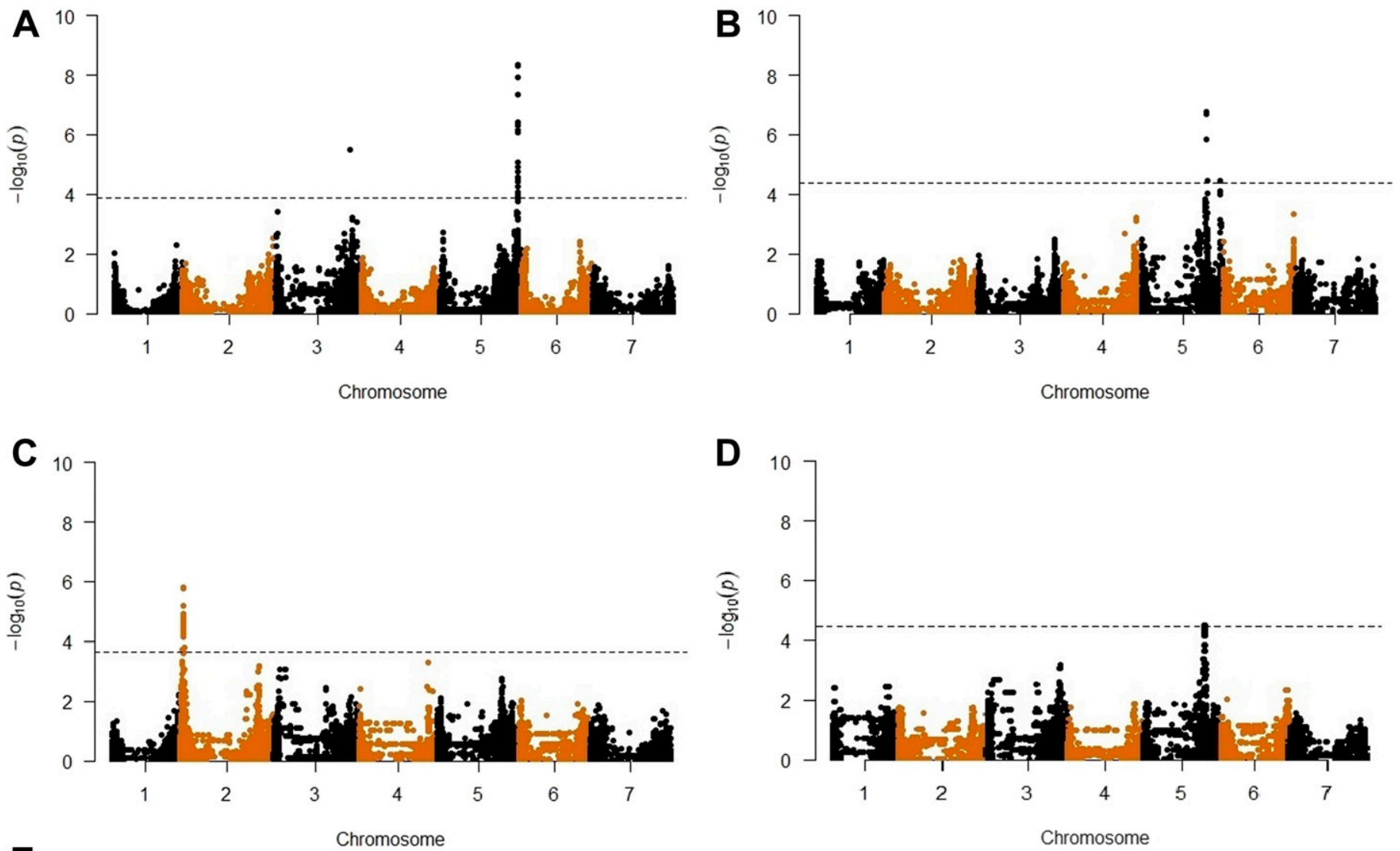

E

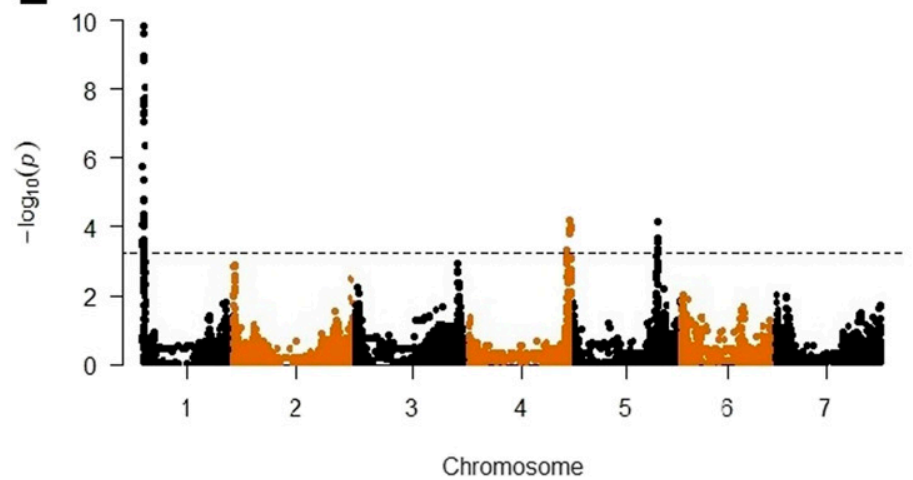

Fig. 3. Manhattan plots from the genome-wide association study (GWAS) of coefficient of infection (CI) values from the Cycle II barley population after infection by Puccinia graminis f. sp. tritici race TTKSK at seedling stage under controlled environment conditions, A, disease severity values after infection by $P$. graminis $\mathrm{f}$. sp. tritici race QCCJB at the adult plant stage under field conditions in B, 2018 and $\mathbf{C}$ and D, 2019, and $\mathbf{E}$, best linear unbiased predictions (BLUPs) for disease severity after infection by P. striiformis f. sp. hordei in the Cycle II barley population across four environments (Corvallis 2018-2019 and Davis 2018-2019) at adult plant stage. Panel C shows the GWAS Manhattan plot of all phenotypic data from 2019 and panel D shows the GWAS Manhattan plot of phenotype data from Cycle II lines with heading dates $\geq 59$ days after planting. 
resistance gene/QTL introgressions were selected based on their breeding potential (elite material from OSU) and the possibility of pyramiding new sources of stem rust resistance from UC-Davis material. The presence of a QTL for resistance at the seedling stage on $5 \mathrm{H}$ in the Cycle I selections was inferred from the allele type at the most significant SNP.

Unexpectedly, three lines from the OSU barley program that were thought to be susceptible (Thunder, DH120412, and DH130939) exhibited a resistant reaction at the seedling stage. Additionally, potential new sources of stem rust resistance were confirmed in the UC-Davis material. The lines from OSU and UC-Davis that did not amplify for Rpg 5 but exhibited resistance at the seedling stage are potentially new sources of resistance. The lack of segregation in progeny of crosses between Rpg5-positive parents and those where no amplification was observed leads us to hypothesize that an alternative allele at Rpg5, or at a tightly linked locus, is involved. Further studies will be required to design and validate a new set of markers.

As reported by Hernandez et al. (2019) and Sharma Poudel et al. (2018), the rpg4/Rpg5 complex is necessary but not sufficient to confer resistance. Hernandez et al. (2019) found that QTL5 and QTL7 are required to confer resistance in lines carrying Rpg5. Sharma Poudel et al. (2018) found that locus Rrrl (required for rpg4-mediated resistance 1) is required along with the rpg4/Rpg5 complex to confer resistance. In this study, neither QTL5 nor QTL7 were found to be associated with resistance at the seedling stage. However, QTL3 was found to be interacting with Rpg5, as lines carrying that combination expressed a significantly lower level of disease compared with lines carrying only Rpg5. The requirement of other elements to achieve higher levels of resistance in the presence of Rpg 5 is confirmed by lines carrying the Rpg 5 resistance allele but exhibiting a susceptible reaction at seedling stage. Previous studies have also detected resistance in this region on $3 \mathrm{H}$. Sallam et al. (2017) found a QTL on $3 \mathrm{H}$ close to this region conferring resistance to stem rust race MCCFC. At the adult stage, Case et al. (2018a) found a significant QTL $\sim 8.19 \mathrm{cM}$ distal associated with resistance to stem rust race QCCJB. Although those detected QTLs were defined as minor QTLs, they may play key roles in defense.

To effectively incorporate different sources of resistance, resistant germplasm identified at the seedling growth stage must be confirmed at the adult plant stage (Gyawali et al. 2018; Park 2008; Singh et al. 2015). For this reason, genotypes evaluated for stem rust at the seedling stage were also tested under field conditions in MN18 and MN19. These experiments confirmed the utility of race QCCJB as a surrogate to detect the rpg4/Rpg5 resistance complex, when it is not possible to use race TTKSK. The surrogate race, however, may not respond the same as race TTKSK
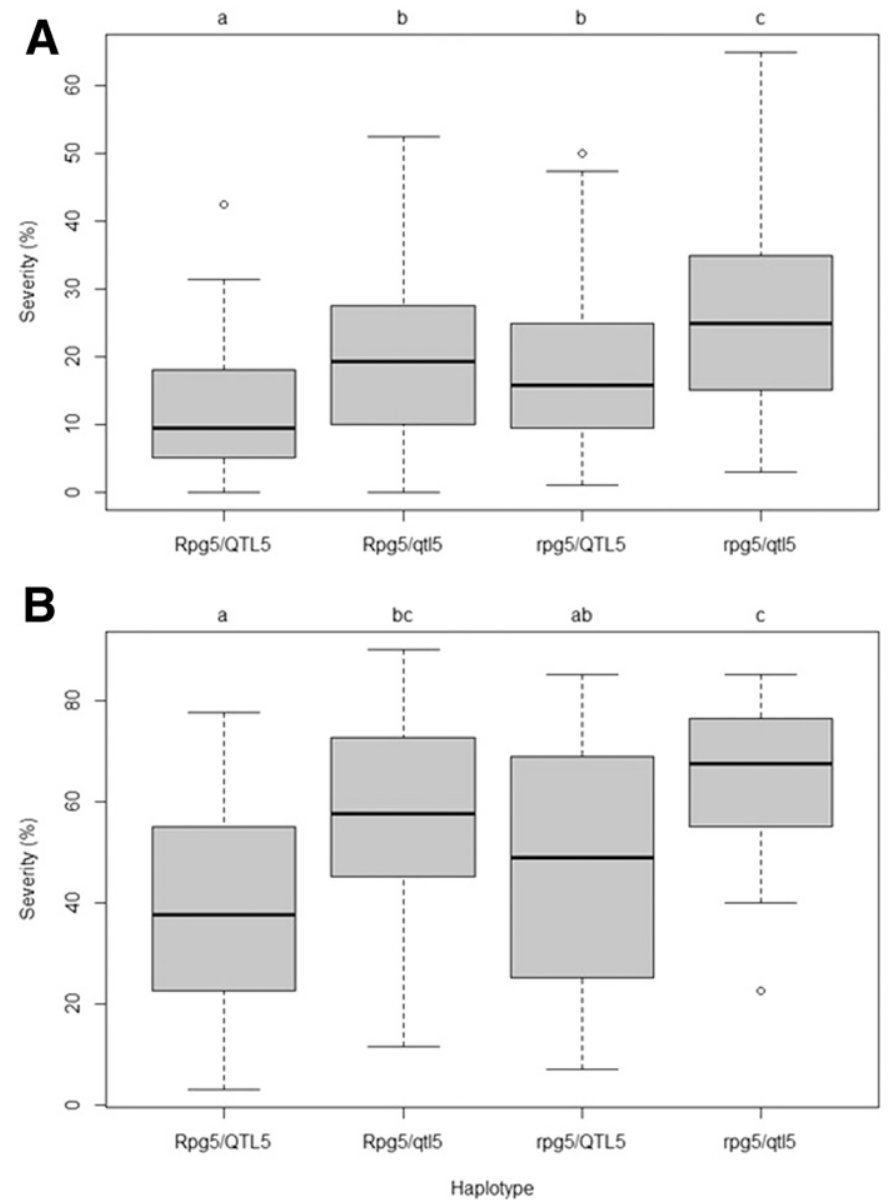

Fig. 4. Box plots for the four two-locus haplotypes (alleles Rpg5 and QTL5) based on disease severity values for the Cycle II barley population after infection by Puccinia graminis f. sp. tritici race QCCJB in A, MN18 and B, MN19HD59. MN19H59 refers to the subset of lines with heading dates $\geq 59$ days after planting in 2019. Means for haplotypes followed by the same letter are not significantly different according to Tukey's honestly significant test $(P>0.05)$.

TABLE 3. Single nucleotide polymorphism (SNP) markers significantly associated with resistance to stem rust and stripe rust in the Cycle II barley population.

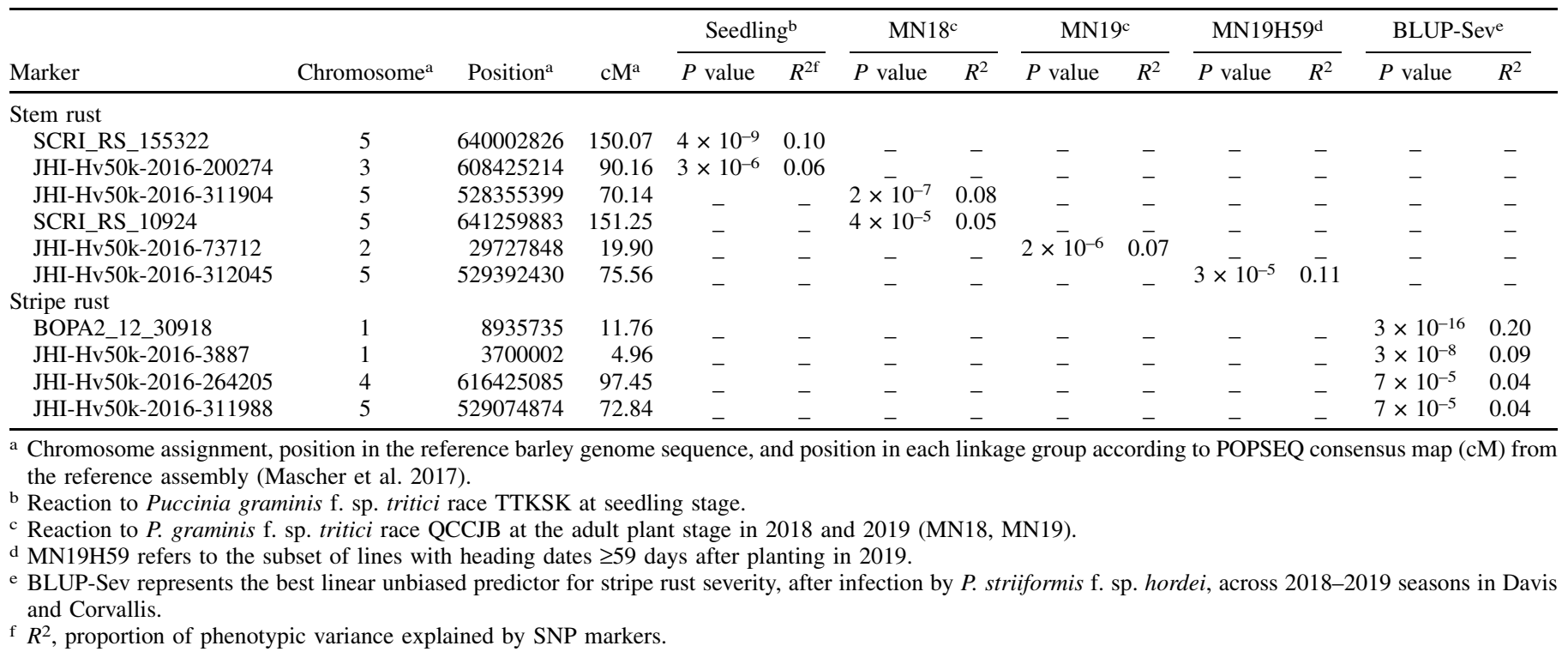


to other genetic factors conferring resistance. Based the adult plant phenotype data from 2018, the $r p 4 / R p g 5$ complex was confirmed as an important and necessary source of resistance to stem rust. However, this complex alone was not sufficient to reduce disease symptoms to an acceptable level: 27 lines with Rpg 5 had disease severities of $\geq 30 \%$. Hernandez et al. (2019) found that the highest level of seedling stage resistance was found when Rpg5 was combined with resistance alleles at QTL5 and QTL7. Although QTL7 was not detected in MN18, a QTL on 5H was found to be associated with high levels of resistance at the adult plant stage in Cycle II. This QTL, however, is over $10 \mathrm{cM}$ distal to the QTL conferring resistance at the seedling stage reported by Hernandez et al. (2019). Using a diverse panel of advanced breeding lines and cultivars from 10 U.S. barley breeding programs, Zhou et al. (2014) reported that $11 \_11355$ at $527152265 \mathrm{Mb}(\sim 72.71 \mathrm{cM})$ had a significant marker association with resistance at the adult stage. Case et al. (2018b) reported the same marker associated with resistance at this growth stage. Moscou et al. (2011), using a biparental population from the cross Q21861 × SM89010, detected a resistance QTL on $5 \mathrm{H}$ at $74.9 \mathrm{cM}$, supporting the notion that this region on $5 \mathrm{H}$ is important for stem rust resistance at the adult stage.

The results of MN19 added an environmental dimension to the assessment of resistance to TTKSK at the adult plant stage, using race QCCJB as a surrogate. In 2019, higher temperatures, which are more likely to occur with climate change (IPCC 2018), resulted in higher disease severities than in 2018. The loss of effectiveness of the rgp4/Rpg5 complex under high temperatures is well documented (Jin et al. 1994; Steffenson et al. 2017; Sun and Steffenson 1997). Confirmation of this temperature sensitivity was provided by the disease phenotype of the resistant control Q21861, which reached 21\% severity in MN19. Furthermore, using MN19 adult plant field data, the rpg4/Rpg5 complex was not detected as a determinant of resistance. This underscores the value of the 5HQTL complex, which remained an effective and significant determinant of resistance under the higher temperatures experienced in 2019. The effects of this QTL are apparent in the GWAS (Fig. 3B and D) and in the two-locus haplotype analysis (Fig. 4), where the importance of the 5H QTL, in both years, is apparent. The 2019 data also revealed the effects of interaction between higher temperature and maturity. Without correction for heading date, the only "resistance" QTL was coincident with $P p d-H 1$, a locus at which the functional allele is associated with accelerated flowering under long-day conditions (Turner et al. 2005). The presence of this dominant allele, combined with higher temperatures at flowering, resulted in earlier maturity and partial disease escape. The QTL on $5 \mathrm{H}$ at cM 75.56 was only detected when the Cycle II GWAS for resistance at the adult plant stage was performed on genotypes with heading dates $\geq 59$ days.

The possible occurrence of multiple resistance alleles in this region $(70.14$ to $75.56 \mathrm{cM})$ of $5 \mathrm{H}$ is supported by the fact that all of the Cycle I parents were resistant in MN18 but only one was resistant in MN19. Haplotype analysis of the progeny from Cycle II, however, did not identify consistent diagnostic patterns for higher resolution analysis of this $\sim 6 \mathrm{cM}$ region. A more thorough understanding of the QTL region located between 70.14 and 75.56 $\mathrm{cM}$ could be facilitated by analysis of a biparental population homozygous for rpg5 but with recombinants in the 5H QTL region at the adult plant stage and under high temperature conditions.

The stripe rust QTL we found on $1 \mathrm{H}$ is described in the literature. Using a doubled haploid population derived from the cross of Shyri and Galena, Toojinda et al. (2000) mapped a stripe rust resistance QTL on the short arm of $1 \mathrm{H}$ explaining 28 to $50 \%$ of phenotypic variation from adult stage field data. The parent Shyri contributed this resistance allele. Based on the same population but with phenotypic values derived at the seedling stage, Castro et al. (2002b) identified a major QTL associated with resistance to three P. striiformis f. sp. hordei races (PSH1, PSH13, and PSH14). This QTL is coincident with the QTL reported by Toojinda et al. (2000). Richardson et al. (2006) used marker assisted selection to introgress and validate this major QTL into the susceptible genetic background of cultivar Baronesse. We confirmed this major effect QTL in our Cycle II population, which expressed a large effect for resistance at the adult plant stage across locations. The resistance locus found on $4 \mathrm{H}$ has also been previously described in different populations. Using Calicuchima-sib as a source of resistance, Chen et al. (1994) found a QTL on $4 \mathrm{H}$ associated with stripe rust resistance at the adult stage. Castro et al. (2002b) used the same population and a larger set of markers to validate resistance from Calicuchima-sib for seedling and adult plant stages at the same genomic region. This QTL was further introgressed into the susceptible background of cultivar Harrington, which confirmed the resistance effects of the $4 \mathrm{H}$ locus. Esvelt Klos et al. (2016) identified a major QTL on $4 \mathrm{H}$ conferring resistance at the seedling and adult stages using a biparental population from cross Lenetah $\times$ GZ. Calicuchima-sib was also the source of resistance found on $5 \mathrm{H}$ at the seedling and adult plant stage. Chen et al. (1994) and Castro et al. (2002a) found a significant QTL on $5 \mathrm{H}$ chromosome explaining 57 and $67.8 \%$ of phenotypic variation, respectively. The former Mexico-based ICARDA/CIMMYT program, under the direction of the late Dr. Hugo Vivar, was the source of the stripe rust resistance QTLs on chromosomes 1H, 4H, and 5H (Chen et al. 1994; Toojinda et al. 2000) in the OSU program. These QTL alleles have remained effective against prevailing races in the United States since their first deployment 25 years ago. In this report, we confirm the

TABLE 4. Selected doubled haploids from Cycle II barley population with resistance to Puccinia graminis f. sp. tritici race TTKSK at seedling stage and race QCCJB at the adult plant stage and P. striiformis f. sp. hordei at adult plant stage

\begin{tabular}{llccccccc}
\hline Line & \multicolumn{1}{c}{ Pedigree } & IT-M $^{\mathrm{a}}$ & MN18 $^{\mathrm{b}}$ & MN19HD59 $^{\mathrm{c}}$ & BLUP-Sev $^{\mathrm{d}}$ & Row type & Hull type $^{\text {Vernalization requirement }^{\mathrm{e}}}$ \\
\hline DH160419 & UC1266/DH140213 & $0 ; 1$ & 6.0 & 8.5 & 6.4 & 6 & Naked & V- \\
DH160733 & DH140512/UC1322 & $0 ; 1-$ & 4.0 & 6.0 & 2.5 & 2 & Covered & C- \\
DH160734 & DH140512/UC1322 & $0 ;$ & 6.5 & 15.0 & 2.5 & 2 & Covered & V- \\
DH160745 & DH140512/UC1322 & $0 ;$ & 5.0 & 11.5 & 2.6 & 2 & Covered & V- \\
DH160748 & DH140512/UC1322 & $0 ;$ & 2.0 & 3.0 & 2.6 & 2 & Covered & V- \\
DH160754 & DH140512/UC1322 & $0 ;$ & 1.0 & 7.0 & 2.0 & 2 & Covered & V- \\
DH161043 & DH140512/UC1322 & $0 ; 1$ & 3.0 & 3.0 & 2.5 & 2 & Covered & V- \\
DH161921 & DH140512/DH130004 & $0 ; 1$ & 11.0 & 12.5 & 3.5 & 2 & Covered & V- \\
DH161926 & DH140512/DH130004 & $0 ; 1$ & 4.0 & 6.0 & 8.1 & 2 & Covered & V- \\
DH161927 & DH140512/DH130004 & $0 ; 1$ & 12.5 & 15.0 & 7.5 & 2 & Covered & V- \\
DH161914 & DH140512/10.0860 & $0 ; 1-$ & 5.0 & 7.0 & 3.1 & 2 & Covered & V- \\
DH160779 & DH140030/UC1231L & $10 ;$ & 2.0 & 5.0 & 4.2 & 6 & Naked & V- \\
\hline
\end{tabular}

a Infection type mode (IT-M) after infection by $P$. graminis f. sp. tritici race TTKSK.

b Adult plant stage severity after infection by $P$. graminis f. sp. tritici race QCCJB in 2018.

c MN19HD59 refers to DH progeny in 2019 with heading dates $\geq 59$ days after planting.

${ }^{\mathrm{d}}$ BLUP-Sev represents the best linear unbiased predictor for stripe rust severity after infection by $P$. striiformis $\mathrm{f}$. sp. hordei, across 2018-2019 seasons in Davis and Corvallis.

e Lines without ( $\mathrm{V}-$ ) vernalization requirements based on greenhouse evaluation under controlled conditions. 
continued effectiveness of these resistance QTLs at the adult stage in recent years and at representative test sites in Oregon and California. The range of races used to inoculate these trials confirms the nonrace specificity of these resistance loci. Furthermore, the BLUPs used to detect significant genomic regions associated with resistance is a robust analysis that confirms the effect of these QTL across different environments. As a whole, pyramiding multiple resistance genes improves durable resistance against BSR. However, the lack of new resistance genes/QTLs is a concern.

The Mexico-based ICARDA/CIMMYT program was also a likely source of the rpg4/Rpg5 complex locus discovered in Q21861 (DillMacky et al. 1992; Jin et al. 1994). In this report, we confirm the necessity of this locus for resistance, at the adult plant stage, to stem rust, as well as its insufficiency. Additional factors are necessary for resistance, including one or more of the QTLs discovered at the seedling and adult plant stages. Interestingly, the stripe rust resistance QTL on chromosome $5 \mathrm{H}$ is in the same $\sim 6 \mathrm{cM}$ interval as the QTL(s) associated with resistance to stem rust at the adult plant stage. Genes/QTLs conferring resistance to multiple specificities of the same pathogen, or to different pathogens, have been reported (Chen et al. 2003; Inukai et al. 2006). These effects can be due to tight linkage or pleiotropy. The purposeful introgression of the 5H BSR QTL into OSU and UC-Davis germplasm may have inadvertently led to the introgression of QTL(s) for resistance to stem rust at the adult plant stage and an additional safeguard against stem rust under high temperature conditions. The resistant germplasm described in Table 4 is a resource available to the research and breeding communities. It was developed using abundant genome-wide markers to map and validate genes/QTL conferring resistance to the two pathogens. These genotyping tools, or derivatives of them, will be useful in systematically and efficiently pyramiding resistance genes/QTL and selecting for other economically important traits in new germplasm.

Conclusions. We have expanded the germplasm base of adapted barley germplasm resistant to TTKSK, beginning with introgression of the rpg4/Rpg5 complex from the invaluable genetic stock Q21861, and culminating with the additional introgression of a QTL on chromosome $5 \mathrm{H}$, which continues to confer resistance under higher temperature conditions. Higher temperature conditions at flowering are increasingly likely with climate change. The lack of Rpg5 amplification in "susceptible" parents chosen as recipients for introgression of the rpg4/ Rpg 5 complex and the absence of segregation at seedling stage in crosses of this germplasm with known carriers of rpg4/Rpg 5 suggests the presence of an alternative allele at Rpg5 or at a tightly linked locus. Research is underway to determine the basis of Rpg5Xx. We have validated the continued effectiveness of the BSR resistance QTLs on $1 \mathrm{H}$, $4 \mathrm{H}$, and $5 \mathrm{H}$ and identified a potential overlap of resistance to the two rusts on chromosome $5 \mathrm{H}$. Research is underway to develop a deeper understanding of the genetic architecture of this important region.

\section{ACKNOWLEDGMENTS}

We thank Shiaoman Chao (retired) USDA-ARS Fargo, North Dakota for Illumina $50 \mathrm{~K}$ genotyping and allele calling, Rick Gadzinski and Sean Tracey at Functional Biosciences for genotyping related to Rpg5, and Austin Case for excellent technical assistance and phenotyping for TTKSK in the Biosafety Level 3 Containment Facility at the University of Minnesota in St. Paul.

\section{LITERATURE CITED}

Ali, S., Gladieux, P., Leconte, M., Gautier, A., Justesen, A. F., Hovmøller, M. S., Enjalbert, J., and de Vallavieille-Pope, C. 2014. Origin, migration routes and worldwide population genetic structure of the wheat yellow rust pathogen Puccinia striiformis f. sp. tritici. PLoS Pathog. 10:e1003903.

Alves, M. S., Dadalto, S. P., Gonçalves, A. B., De Souza, G. B., Barros, V. A., and Fietto, L. G. 2013. Plant bZIP transcription factors responsive to pathogens: A review. Int. J. Mol. 14:7815-7828.

Arora, D., Gross, T., and Brueggeman, R. 2013. Allele characterization of genes required for $r p g 4$-mediated wheat stem rust resistance identifies Rpg5 as the R gene. Phytopathology 103:1153-1161.
Badr, A., Muller, K., Schafer, R., El Rabey, H., Effgen, S., Ibrahim, H. H., Pozzi, C., Rohde, W., and Salamini, F. 2000. On the origin and domestication history of barley (Hordeum vulgare). Mol. Biol. Evol. 17:499-510.

Bayer, M. M., Rapazote-Flores, P., Ganal, M., Hedley, P. E., Macaulay, M., Plieske, J., Ramsay, L., Russell, J., Shaw, P. D., Thomas, W., and Waugh, R. 2017. Development and evaluation of a barley 50k iSelect SNP array. Front. Plant Sci. 8:1792.

Belcher, A. R., Cuesta-Marcos, A., Smith, K. P., Mundt, C. C., Chen, X., and Hayes, P. M. 2018. TCAP FAC-WIN6 elite barley GWAS panel QTL. I. Barley stripe rust resistance QTL in facultative and winter six-rowed malt barley breeding programs identified via GWAS. Crop Sci. 58:103-119.

Benjamini, Y., and Hochberg, Y. 1995. Controlling the false discovery rate: A practical and powerful approach to multiple testing. J. R. Stat. Soc. B 57: 289-300.

Bradbury, P. J., Zhang, Z., Kroon, D. E., Casstevens, T. M., Ramdoss, Y., and Buckler, E. S. 2007. TASSEL: Software for association mapping of complex traits in diverse samples. Bioinformatics 23:2633-2635.

Cantalapiedra, C. P., Boudiar, R., Casas, A. M., Igartua, E., and Contreras-Moreira, B. 2015. BARLEYMAP: Physical and genetic mapping of nucleotide sequences and annotation of surrounding loci in barley. Mol. Breed. 35:13.

Case, A. J., Bhavani, S., Macharia, G., Pretorius, Z., Coetzee, V., Kloppers, F., Tyagi, P., Brown-Guedira, G., and Steffenson, B. J. 2018a. Mapping adult plant stem rust resistance in barley accessions Hietpas-5 and GAW-79. Theor. Appl. Genet. 131:1-22.

Case, A. J., Bhavani, S., Macharia, G., and Steffenson, B. J. 2018b. Genomewide association study of stem rust resistance in a world collection of cultivated barley. Theor. Appl. Genet. 131:107-126.

Castro, A., Capettini, F., Corey, A., Filichkina, T., Hayes, P., Kleinhofs, A., Kudrna, D., Richardson, K., Sandoval-Islas, S., Rossi, C., and Vivar, H. 2003a. Mapping and pyramiding of qualitative and quantitative resistance to stripe rust in barley. Theor. Appl. Genet. 107:922-930.

Castro, A., Hayes, P. M., Fillichkin, T., and Rossi, C. 2002a. Update of barley stripe rust resistance QTL in the Calicuchima-sib $\times$ Bowman mapping population. Barley Genet. Newsl. 32:1-12.

Castro, A. J., Chen, X., Hayes, P. M., and Johnston, M. 2003b. Pyramiding quantitative trait locus (QTL) alleles determining resistance to barley stripe rust. Crop Sci. 43:651-659.

Castro, A. J., Chen, X., Hayes, P. M., Knapp, S. J., Line, R. F., Toojinda, T., and Vivar, H. 2002b. Coincident QTL which determine seedling and adult plant resistance to stripe rust in barley. Crop Sci. 42:1701-1708.

Chen, F., Prehn, D., Hayes, P., Mulrooney, D., Corey, A., and Vivar, H. 1994. Mapping genes for resistance to barley stripe rust (Puccinia striiformis $\mathrm{f}$. sp. hordei). Theor. Appl. Genet. 88:215-219.

Chen, H., Wang, S., Xing, Y., Xu, C., Hayes, P. M., and Zhang, Q. 2003. Comparative analyses of genomic locations and race specificities of loci for quantitative resistance to Pyricularia grisea in rice and barley. Proc. Natl. Acad. Sci. USA 100:2544-2549.

Chen, X. 2005. Epidemiology and control of stripe rust [Puccinia striiformis $\mathrm{f}$. sp. tritici] on wheat. Can. J. Plant Pathol. 27:314-337.

Chen, X., and Kang, Z. 2017. Stripe Rust. Springer, Netherlands.

Chen, X., and Line, R. F. 1999. Recessive genes for resistance to Puccinia striiformis f. sp. hordei in barley. Phytopathology 89:226-232.

Chen, X., and Line, R. F. 2003. Identification of genes for resistance to Puccinia striiformis f. sp. hordei in 18 barley genotypes. Euphytica 129:127-146.

Chen, X., Line, R. F., and Leung, H. 1995. Virulence and polymorphic DNA relationships of Puccinia striiformis $\mathrm{f}$. sp. hordei to other rusts. Phytopathology 85:1335-1342.

Comadran, J., Kilian, B., Russell, J., Ramsay, L., Stein, N., Ganal, M., Shaw, P., Bayer, M., Thomas, W., Marshall, D., Hedley, P. E., Tondelli, A., Pecchioni, N., Francia, E., Korzun, V., Walther, A., and Waugh, R. 2012. Natural variation in a homolog of Antirrhinum CENTRORADIALIS contributed to spring growth habit and environmental adaptation in cultivated barley. Nat. Genet. 44:1388-1392.

Dean, R., Van Kan, J. A. L., Pretorius, Z., Hammond-Kosack, K., Di Pietro, A., Spanu, P. D., Rudd, J. J., Dickman, M., Kahmann, R., Ellis, J., and Foster, G. D. 2012. The top 10 fungal pathogens in molecular plant pathology. Mol. Plant Pathol. 13:414-430.

Derevnina, L., Fetch, T., Singh, D., Brueggeman, R., Dong, C., and Park, R. 2014. Analysis of stem rust resistance in Australian barley cultivars. Plant Dis. 98:1485-1493.

Digel, B., Tavakol, E., Verderio, G., Tondelli, A., Xu, X., Cattivelli, L., Rossini, L., and von Korff, M. 2016. Photoperiod-H1 (Ppd-H1) controls leaf size. Plant Physiol. 172:405-415.

Dill-Macky, R., Rees, R., and Boyd, W. 1992. Sources of resistance to stem rust in barley. Plant Dis. 76:212.

Dubin, H., and Stubbs, R. 1986. Epidemic spread of barley stripe rust in South America. Plant Dis. 70:141-144.

Esvelt Klos, K., Gordon, T., Bregitzer, P., Hayes, P., Chen, X., Del Blanco, I., Fisk, S., and Bonman, J. 2016. Barley stripe rust resistance QTL: 
Development and validation of SNP markers for resistance to Puccinia striiformis f. sp. hordei. Phytopathology 106:1344-1351.

FAOSTAT. 2017. Production and Trade. Food and Agriculture Organization of the United Nations. FAO, Rome, Italy.

Goff, K. E., and Ramonell, K. M. 2007. The role and regulation of receptorlike kinases in plant defense. Gene Regul. Syst. Bio. 1:167-175.

Gutiérrez, L., Germán, S., Pereyra, S., Hayes, P. M., Pérez, C. A., Capettini, F., Locatelli, A., Berberian, N. M., Falconi, E. E., Estrada, R., Fros, D., Gonza, V., Altamirano, H., Huerta-Espino, J., Neyra, E., Orjeda, G., Sandoval-Islas, S., Singh, R., Turkington, K., and Castro, A. J. 2015. Multi-environment multi-QTL association mapping identifies disease resistance QTL in barley germplasm from Latin America. Theor. Appl. Genet. 128:501-516.

Gyawali, S., Verma, R. P. S., Kumar, S., Bhardwaj, S. C., Gangwar, O. P., Selvakumar, R., Shekhawat, P. S., Rehman, S., and Sharma-Poudyal, D. 2018. Seedling and adult-plant stage resistance of a world collection of barley genotypes to stripe rust. J. Phytopathol. 166:18-27.

Hayes, P., Castro, A. L., Corey, A., Henson, C., Jones, B. L., Kling, J. G., Mather, D., Matus, I., Rossi, C., and Sato, K. 2003. Genetic diversity for quantitatively inherited agronomic and malting quality traits. Page 201 in: Diversity in Barley (Hordeum vulgare). R. Von Bothmer, H. Knupffer, T. van Hintum, and K. Sato, eds. Elsevier Science Publishers, Amsterdam.

Hernandez, J., Steffenson, B. J., Filichkin, T., Fisk, S. P., Helgerson, L., Meints, B., Vining, K. J., Marshall, D., del Blanco, A., Chen, X., and Hayes, P. M. 2019. Introgression of rpg4/Rpg5 into barley germplasm provides insights into the genetics of resistance to Puccinia graminis f. sp. tritici race TTKSK and resources for developing resistant cultivars. Phytopathology 109:1018-1028

Inukai, T., Vales, M. I., Hori, K., Sato, K., and Hayes, P. M. 2006. RMo1 confers blast resistance in barley and is located within the complex of resistance genes containing Mla, a powdery mildew resistance gene. Mol. Plant-Microbe Interact. 19:1034-1041.

Jin, Y., Steffenson, B., and Miller, J. 1994. Inheritance of resistance to pathotypes QCC and MCC of Puccinia graminis f. sp. tritici in barley line Q21861 and temperature effects on the expression of resistance. Phytopathology 84:452-455

Line, R. F. 2002. Stripe rust of wheat and barley in North America: A retrospective historical review. Annu. Rev. Phytopathol. 40:75-118.

Marshall, D., and Sutton, R. 1995. Epidemiology of stripe rust, virulence of Puccinia striiformis f. sp. hordei, and yield loss in barley. Plant Dis. 79:732-737.

Mascher, M., Gundlach, H., Himmelbach, A., Beier, S., Twardziok, S. O., Wicker, T., Radchuk, V., Dockter, C., Hedley, P. E., Russell, J., Bayer, M., Ramsay, L., Liu, H., Haberer, G., Zhang, X.-Q., Zhang, Q., Barrero, R. A., Li, L., Taudien, S., Groth, M., Felder, M., Hastie, A., Šimková, H., Staňková, H., Vrána, J., Chan, S., Muñoz-Amatriaín, M., Ounit, R., Wanamaker, S., Bolser, D., Colmsee, C., Schmutzer, T., Aliyeva-Schnorr, L., Grasso, S., Tanskanen, J., Chailyan, A., Sampath, D., Heavens, D., Clissold, L., Cao, S., Chapman, B., Dai, F., Han, Y., Li, H., Li, X., Lin, C., McCooke, J. K., Tan, C., Wang, P., Wang, S., Yin, S., Zhou, G., Poland, J. A., Bellgard, M. I., Borisjuk, L., Houben, A., Doležel, J., Ayling, S., Lonardi, S., Kersey, P., Langridge, P., Muehlbauer, G. J., Clark, M. D., Caccamo, M., Schulman, A. H., Mayer, K. F. X., Platzer, M., Close, T. J., Scholz, U., Hansson, M., Zhang, G., Braumann, I., Spannagl, M., Li, C., Waugh, R., and Stein, N. 2017. A chromosome conformation capture ordered sequence of the barley genome. Nature 544:427-433.

McNeal, F., Konzak, C., Smith, E., Tate, W., and Russell, T. 1971. A uniform system for recording and processing cereal research data. U.S. Department of Agriculture-Agricultural Research Service, Washington, DC.

Meints, B., Cuesta-Marcos, A., Fisk, S., Ross, A., and Hayes, P. 2016. Food barley quality improvement and germplasm utilization. Pages 41-73 in: Exploration, Identification and Utilization of Barley Germplasm. Z. Guoping and L. Chengdao, eds. Elsevier, London, UK.

Miller, J. D., and Lambert, J. 1955. Variability and inheritance of reaction of barley to race 15B of stem rust. Agron. J. 47:373-377.

Moscou, M. J., Lauter, N., Steffenson, B., and Wise, R. P. 2011. Quantitative and qualitative stem rust resistance factors in barley are associated with transcriptional suppression of defense regulons. PLoS Genet. 7:e1002208.

Mundt, C. C. 2018. Pyramiding for resistance durability: Theory and practice. Phytopathology 108:792-802.

Muñoz-Amatriaín, M., Cuesta-Marcos, A., Endelman, J. B., Comadran, J., Bonman, J. M., Bockelman, H. E., Chao, S., Russell, J., Waugh, R., Hayes, P. M., and Muehlbauer, G. 2014a. The USDA barley core collection: Genetic diversity, population structure, and potential for genome-wide association studies. PLoS One 9:e94688.

Muñoz-Amatriaín, M., Cuesta-Marcos, A., Hayes, P. M., and Muehlbauer, G. J. 2014b. Barley genetic variation: Implications for crop improvement. Brief. Funct. Genomics 13:341-350.
Park, R. 2008. Breeding cereals for rust resistance in Australia. Plant Pathol. 57:591-602.

Parlevliet, J. 1983. Race-specific resistance and cultivar-specific virulence in the barley-leaf rust pathosystem and their consequences for the breeding of leaf rust resistant barley. Euphytica 32:367-375.

Peterson, R. F., Campbell, A., and Hannah, A. 1948. A diagrammatic scale for estimating rust intensity on leaves and stems of cereals. Can. J. Res. 26:496-500.

R Development Core Team. 2015. R: A Language and Environment for Statistical Computing. R Foundation for Statistical Computing, Vienna, Austria.

Richardson, K., Vales, M., Kling, J., Mundt, C., and Hayes, P. 2006. Pyramiding and dissecting disease resistance QTL to barley stripe rust. Theor. Appl. Genet. 113:485-495.

Roelfs, A. 1985. Wheat and rye stem rust. Pages 3-37 in: Diseases, Distribution, Epidemiology, and Control, Elsevier.

Roelfs, A., Huertaespino, J., and Marshall, D. 1992. Barley stripe rust in Texas. Plant Dis. 76:538.

Sallam, A. H., Tyagi, P., Brown-Guedira, G., Muehlbauer, G. J., Hulse, A., and Steffenson, B. J. 2017. Genome-wide association mapping of stem rust resistance in Hordeum vulgare subsp. spontaneum. G3: Genes, Genomes. Genetics 7:3491-3507.

Sharma Poudel, R., Alhashel, A., Gross, T., Gross, P., and Brueggeman, R. S. 2018. Pyramiding rpg4- and Rpgl-mediated stem rust resistance in barley requires the Rrrl gene for both to function. Front. Plant Sci. 9:1789.

Singh, R. P., Hodson, D. P., Jin, Y., Lagudah, E. S., Ayliffe, M. A., Bhavani, S., Rouse, M. N., Pretorius, Z. A., Szabo, L. J., Huerta-Espino, J., Basnet, B., Lan, C., and Hovmøller, M. S. 2015. Emergence and spread of new races of wheat stem rust fungus: Continued threat to food security and prospects of genetic control. Phytopathology 105:872-884.

Stakman, E., Stewart, D., and Loegering, W. 1962. Identification of physiologic races of Puccinia graminis f. sp. tritici. U.S. Department of Agriculture-Agricultural Research Service E-617.

Steffenson, B., Case, A., Pretorius, Z., Coetzee, V., Kloppers, F., Zhou, H., Chai, Y., Wanyera, R., Macharia, G., Bhavani, S., and Grando, S. 2017. Vulnerability of barley to African pathotypes of Puccinia graminis f. sp. tritici and sources of resistance. Phytopathology 107:950-962.

Steffenson, B., Jin, Y., Brueggeman, R., Kleinhofs, A., and Sun, Y. 2009. Resistance to stem rust race TTKSK maps to the rpg4/Rpg5 complex of chromosome 5H of barley. Phytopathology 99:1135-1141.

Steffenson, B. J. 1992. Analysis of durable resistance to stem rust in barley. Euphytica 63:153-167.

Steffenson, B. J., and Smith, K. P. 2006. Breeding barley for multiple disease resistance in the Upper Midwest region of the USA. Czech J. Genet. Plant Breed. 42:79-86.

Steffenson, B. J., Zhou, H., Chai, Y., and Grando, S. 2013. Vulnerability of cultivated and wild barley to African stem rust race TTKSK. Pages 243-255 in: Advance in Barley Sciences. Proc. 11th Int. Barley Genet. Symp. G. Zhang, C. Li, and X. Liu, eds. Zhejiang University Press, Hangzhou, China.

Sun, Y., and Steffenson, B. J. 1997. Effect of incubation time and temperature on the phenotypic expression of rpg4 to Puccinia graminis f. sp. tritici in barley. Can. J. Plant Pathol. 19:25-29.

Toojinda, T., Broers, L., Chen, X., Hayes, P., Kleinhofs, A., Korte, J., Kudrna, D., Leung, H., Line, R., Powell, W., Ramsay, L., Vivar, H., and Waugh, R. 2000. Mapping quantitative and qualitative disease resistance genes in a doubled haploid population of barley (Hordeum vulgare). Theor. Appl. Genet. 101:580-589.

Tornero, P., Chao, R. A., Luthin, W. N., Goff, S. A., and Dangl, J. L. 2002. Large-scale structure-function analysis of the Arabidopsis RPM1 disease resistance protein. Plant Cell 14:435-450.

Turner, A., Beales, J., Faure, S., Dunford, R. P., and Laurie, D. A. 2005. The pseudo-response regulator $\mathrm{Ppd}-\mathrm{Hl}$ provides adaptation to photoperiod in barley. Science 310:1031-1034.

Vales, M., Schön, C., Capettini, F., Chen, X., Corey, A., Mather, D., Mundt, C., Richardson, K., Sandoval-Islas, J., Utz, H., and Hayes, P. 2005. Effect of population size on the estimation of QTL: A test using resistance to barley stripe rust. Theor. Appl. Genet. 111:1260-1270.

Wang, X., Richards, J., Gross, T., Druka, A., Kleinhofs, A., Steffenson, B., Acevedo, M., and Brueggeman, R. 2013. The rpg4-mediated resistance to wheat stem rust (Puccinia graminis) in barley (Hordeum vulgare) requires Rpg5, a second NBS-LRR gene, and an actin depolymerization factor. Mol. Plant-Microbe Interact. 26:407-418.

Yan, G., and Chen, X. 2006. Molecular mapping of a recessive gene for resistance to stripe rust in barley. Theor. Appl. Genet. 113:529-537.

Zadoks, J. C., Chang, T. T., and Konzak, C. F. 1974. A decimal code for the growth stages of cereals. Weed Res. 14:415-421.

Zhou, H., Steffenson, B., Muehlbauer, G., Wanyera, R., Njau, P., and Ndeda, S. 2014. Association mapping of stem rust race TTKSK resistance in US barley breeding germplasm. Theor. Appl. Genet. 127:1293-1304. 